\title{
EFFECTS OF VIRTUAL REALITY ON PARKINSONIAN GAIT: BLIND CONTROLLED RANDOMIZED CLINICAL TRIAL PROTOCOL
}

\author{
Gileno Edu Lameira de Melo, ${ }^{1,2}$ Renata Calhes Franco de Moura', Jamile Benite Palma Lopes', \\ Paulo Roberto Fonseca Junior', Roberta Delasta Lazzari', Natália de Almeida Carvalho Duarte', \\ Jose Robertto Zaffalon Junior,1,2, Arislander Jonathan Lopes Dumont', Ana Francisca Rozin Kleiner ${ }^{3}$, Manuela Galli³, \\ Luiz Alfredo Braun Ferreira ${ }^{4,5}$, Erika Okamoto ${ }^{6}$, Claudia Santos Oliveira ${ }^{7 *}$
}

\begin{abstract}
Background: Parkinson's disease (PD) is a neurodegenerative condition that exerts a negative influence on postural control and mobility, predominantly among older adults, affecting mobility and increasing the risk of slipping, tripping and falls. Objectives: Determine the effectiveness of gait training with the aid of virtual reality on balance and gait in comparison to treadmill training and conventional gait training for subjects with PD. Methods/design: A prospective, randomized, controlled blind, clinical trial is proposed. The sample will comprise 45 individuals with Parkinson's disease randomly allocated to three groups (Control: conventional gait training; Experimental I: treadmill training; and Experimental II: gait training involving virtual reality). Evaluations will be conducted on four occasions: 1) pre-intervention; 2) immediately after one session; 3) post-intervention; and 4) one month after last session. The evaluations will involve the Berg Balance Scale, Tinetti Test, Unified Parkinson's Disease Rating Scale and Hoehn and Yahr Scale. The G-WALK (BTS) inertial sensor will be used for the evaluation of spatiotemporal gait variables, the Timed Up and Go test and the Six-Minute Walk Test. Training will involve 20-minute sessions held three times per week for four consecutive weeks. The results will be analyzed statistically by two-way ANOVA for the data with parametric distribution or Friedman's two-way ANOVA for non-parametric data $(\alpha<0.05)$. Conclusion: The proposed study will compare gait conventional training, treadmill training and training with virtual reality with regard to improvements in gait and balance in patients with PD. The results will be published and will contribute evidence on the use of these interventions for individuals with PD.
\end{abstract}

Keywords: Parkinson's Disease; Gait; Virtual Reality Exposure Therapy; Balance.

\section{INTRODUCTION}

Parkinson's disease (PD) is a neurodegenerative condition that exerts a negative influence on postural control and mobility, predominantly among older adults, affecting mobility and increasing the risk of slipping, tripping and falls ${ }^{(1)}$. According to Sanfelice ${ }^{(2)}$, the diagnosis of PD in the early phases is not easy, but the classic symptoms of tremor, stiffness and bradykinesis can allow the diagnosis on the first evaluation. Impaired gait and balance in this disease compromise independence and mobility by increasing the risk of falls and associated injuries, with a consequent reduction in quality of life ${ }^{(3)}$.

To promote an improvement in the aspects of gait and the motor rehabilitation process, it is recommended that individuals with PD execute various motor activities, especially the exercise of walking on a treadmil|(4). In studies conducted involving individuals with PD, Mehrholz et al. ${ }^{(5)}$ and
Petzinger et al. ${ }^{(6)}$ report that treadmill training (TT) with and without body weight support is safe and can lead to a more stable gait pattern, thereby improving walking speed and the distance travelled. Moreover, Zettergren ${ }^{(7)}$ states that the use of virtual reality has been gaining ground in the treatment of patients with PD, since interactive videogames require the use of simultaneous physical, visual, auditory, cognitive, psychological and social strategies that can lead to an improvement in functional performance, especially gait.

Studies report that the use of virtual reality for balance and gait training as well as reducing the risk of falls in elderly individuals and patients with neurological diseases has demonstrated positive effects on gait velocity and step length as well as the ability to perform dual tasks and overcome obstacles in comparison to conventional balance training.

Trial registration: This study received approval from the Human Research Ethics Committee of University Nove de Julho (Sao Paulo, Brazil) under process number 1.487.213/2016 and is registered with the Brazilian Registry of Clinical Trials - ReBEC ( $n^{\circ}$ RBR - 8tbhyr) registered on 20 June 2016.

*Correponding author: Claúdia Santos Oliveira. Address: Rua Itapicuru, 380. Apto: 111. Perdizes. CEP: 05006-000, São Paulo, SP, Brazil. Email: csantos@uni9.pro.br. Fone: +1138681681

${ }^{7}$ Professor, Master and Doctoral Programs in Rehabilitation Sciences, Movement Analysis Lab, Universidade Nove de Julho, São Paulo, SP, Brazil. Email: csantos@uni9.pro.br

Full list of author information is available at the end of the article.

Financial Support: This study protocol received no funding.

Submission date 06 August 2017; Acceptance date 11 October 2017; Publication date 14 November 2017 
However, few studies have reported the effectiveness of this modality specifically for patients with $\mathrm{PD}^{(8-10)}$.Galna et al. ${ }^{(11)}$ conducted a study with the aim of developing a computer game to improve dynamic postural control using the $\mathrm{Xbox}$ 360 with Kinect for individuals with PD. The game provided dynamic postural control training through multidirectional tasks with 12 levels of complexity. Mendes et al. ${ }^{(12)}$ employed virtual reality using the Xbox 360 with Kinect, which proved viable for patients with PD, whose performances improved with the progression of the sessions and the gains were maintained 60 days after the end of treatment.

Different traditional treatments are employed for the rehabilitation of individuals with $\mathrm{PD}$, including over ground gait training and, in some studies, treadmill training. However, traditional gait training generally does not lead to the complete recovery of the normal gait pattern. In this context, the use of virtual reality for balance and gait training as well as reducing the risk of falls in individuals with neurological disease has demonstrated positive effects on walking speed, step length, the ability to perform dual tasks and overcoming obstacles in comparison to conventional balance training ${ }^{(8,9,10)}$.

Thus, comparisons of the effects of gait training over the ground, on a treadmill and with virtual reality are of interest to researchers and clinicians who employ these methods. It is therefore pertinent to determine the effects of each of these modalities on gait and balance in individuals with PD.

\section{METHODS/DESIGN}

\section{Primary objective}

Determine the efficacy of an intervention involving gait training with virtual reality with regard to balance and gait in patients with PD in comparison to gait training on a treadmill and over ground.

\section{Hypothesis 1}

Gait training with virtual reality is more effective than training on a treadmill or over ground with regard to improving gait and dynamic balance in patients with PD.

\section{Study design}

A randomized, controlled, blind, clinical trial will be conducted. The protocol for the proposed study is registered with the Brazilian Registry of Clinical Trials ReBEC: RBR8TBHYR.

\section{Ethics approval and consent to participate}

The proposed study will be conducted in compliance with the principles of the Declaration of Helsinki as well as the regulating norms and guidelines for research involving human subjects stipulated by the National Board of Health of the Brazilian Health Ministry issued under code number 466/12. The study received approval from the Human Research Ethics Committee of University Nove de Julho (São Paulo, Brazil) under process number 1.487.213/2016. All participants will receive clarifications regarding the procedures of the study and those who agree to participate voluntarily will sign a statement of informed consent. Participants will have access to all information and will be allowed to withdraw from the study at any time with no negative repercussions.

\section{Recruitment and sample selection}

Patients with PD will be recruited from the Parkinson Association Brazil (Sao Paulo, Brazil) and will be selected based on the following eligibility criteria:

\section{Inclusion criteria:}

- Diagnosis of idiopathic PD in phase 1,2 or 3 on the Hoehn and Yahr scale;

- Score $\geq 46$ on Berg Balance Scale- BBS;

- Adequate vision (with or without glasses);

- Adequate hearing (with or without a hearing aid).

\section{Exclusion criteria:}

- Physical disability;

- Severe heart disease;

- History of seizure;

- Uncontrolled hypertension.

\section{Sample size}

The sample size was calculated based on a study by Ganesan et al. ${ }^{(13)}$. Functional balance measured using the Berg scale was considered for the calculation due to the fact that this scale represents an important clinical outcome and is directly related to the proposed intervention. The study cited was chosen because it involved a similar population to that of the present project (patients with PD). Considering the means of the control group and treadmill training group after four weeks (48.9with a standard deviation of 2.8 in the control group and 51.4 with a standard deviation of 2.0 in the treadmill group), a bidirectional alpha of 0.05 and an $80 \%$ test power, 12 patients would be needed for each group. The sample will be increased by $20 \%$, leading to 15 patients in each group (total: 45 participants).

\section{Randomization and Concealment}

Patients with PD will be randomly allocated to one of three study groups using a block randomization method:

- Control group: over ground treadmill training;

- Treadmill group: gait training on a treadmill; 
- Virtual reality group: gait training with virtual reality.

Block randomization will involve an allocation sequence generated for each block using a random numbers table. The allocation will be sealed in sequentially numbered opaque envelopes. After the pre-intervention evaluation, the participants will be allocated to a given group by opening an envelope. To ensure the blinding of the examiner, this process will be performed by a member of the research team who will not be involved in the recruitment or development of the study. A set of numbered, sealed, opaque envelopes will be used to ensure allocation concealment. Each envelope will contain a card stipulating to which group the patient will be allocated. The Figure 1 displays the flowchart of the recruitment process, patient selection and dropouts.

\section{Evaluations and follow-up}

After the selection of the participants, four evaluations will be performed by two physiotherapists and one physical educator experienced with the evaluation procedures, who will be blinded to the allocation of the participants to the different groups. The four evaluations will be performed in the following order:

Evaluation 1: pre-intervention;

Evaluation 2: after the first training session;

Evaluation 3: after the 12 training sessions;

Evaluation 4: one month after the last training session;

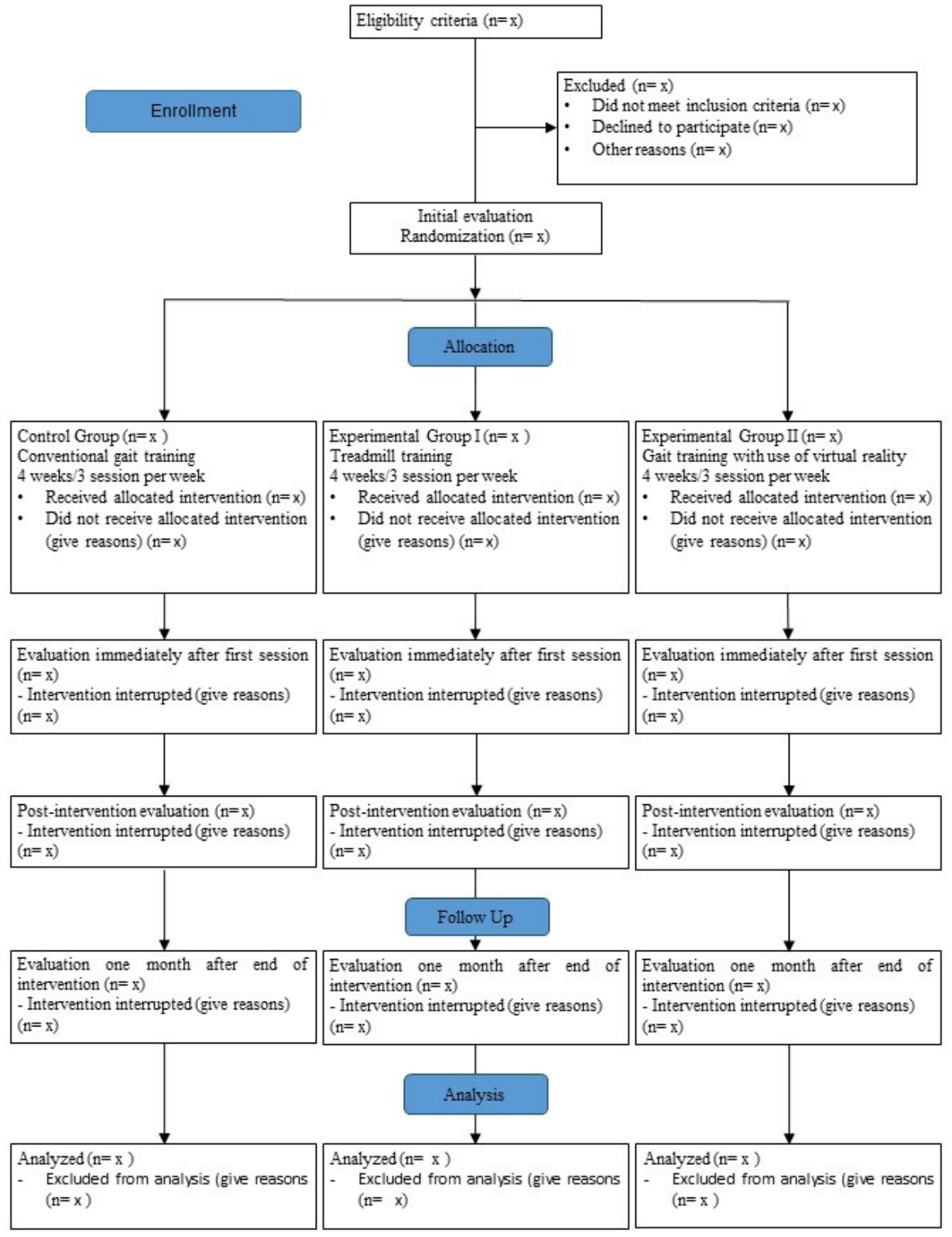

Figure 1. Flowchart of study based on Consolidated Standards of Reporting Trials (CONSORT). 
The assessment tools will be administered on two non-consecutive days, with a maximum of one hour and thirty minutes per day.

\section{Evaluation of functional balance}

- Berg Balance Scale (BBS) -This scale was validated for use in Brazil by Miyamoto et al. ${ }^{(14)}$ and is employed for the evaluation of functional balance. For such, the participant is scored regarding a set of 14 tasks on a five-point scale. The score for each task ranges from 0 (unable to perform the task) to 4 (performs the task independently). The final score ranges from 0 to 56 points, with lower scores denoting a greater risk of falls and higher scores denoting better performance. The cutoff point for the risk of falls is less than 45 points.

- Tinetti test-This test is used to evaluate balance and abnormalities with regard to gait. The test consists of 16 items, nine of which address balance and seven address gait. The Tinetti test classifies aspects of gait, such as velocity, step length, standing symmetry and balance and turning around as well as the changes that occur when the eyes are closed. The tasks are scored from 0 to 1 or from 0 to 2, with a lower score indicating poorer physical ability. The total is determined by the sum of the balance and gait sections. The maximum scores are 16 points for balance and 12 points for gait. Thus, the maximum total score is 28 points. ${ }^{(15)}$

\section{Functional evaluation and severity}

- Unified Parkinson's Disease Rating Scale (UPDRS) - This scale is used for the evaluation of signs and symptoms of the disease as well as the performance of particular activities based on self-reports and clinical observations. The scale is composed of 42 items divided into four parts: 1) mental activity, behavior and mood; 2) activities of daily living; 3 ) motor evaluation; and 4) complications of medicinal therapy. The score for each item ranges from 0 (normal) to 4 (severe) points, with higher scores denoting more impairment due to the disease. ${ }^{(15)}$

- Hoehn and Yahr (H\&Y) Scale -- This scale addresses five stages to evaluate the severity of PD using essentially global measures of signs and symptoms (postural instability, stiffness, tremor and bradykinesia) that allow the classification of the degree of disability. Patients classified in stages I, II and III have mild to moderate disability and those in stage IV and $\mathrm{V}$ have more severe disability ${ }^{(16,17)}$.

\section{Evaluation of gait}

A portable, wireless, inertial sensor system (G-WALK, Gsensor, BTS Bioengenharia SpA, Italy) will be used for the evaluation of spatiotemporal variables of gait as well as the Timed Up and Go (TUG) test and Six-Minute Walk Test (6MWT). This device furnishes linear accelerations on three orthogonal axes: anteroposterior, mediolateral and vertical. The portable Gsensor is composed of a wireless network of inertial sensors for the analysis of human movement. The sensors are controlled by a data recording unit (up to 16 elements) through ZigBee radio communication. The acceleration data will be transmitted via Bluetooth to a computer and processed using the software program designed for the system (BTS G-STUDIO, version 2.6.12.0), which automatically furnishes the parameters ${ }^{(18,19)}$.

For the acquisition of the gait variables, the sensor will be connected to the participant's waist with a semi-elastic strap covering the intervertebral space between $L 4$ and $L 5^{(19)}$. During the walk test, the participant will be instructed to walk at his/her individualized normal pace over a pre-established track. Thus, typical spatiotemporal gait variables will be collected based on the acceleration signals:

(1) Step length $[\mathrm{m}]$ : distance between initial contact of one foot and initial contact of the contralateral foot;

(2) Stride length [m]: distance between two consecutive contacts of the heel of the same foot with the floor;

(3) Step length/height [\%]: step length normalized by the height of the individual;

(4) Velocity $[\mathrm{m} / \mathrm{s}]$ : mean instantaneous velocity within the gait cycle with the integration of acceleration;

(5) Cadence [steps/min]: number of steps per minute;

(6) Propulsion $\left[\mathrm{m} / \mathrm{s}^{2}\right]$ : peak anteroposterior acceleration during swing phase of lower limbs.

\section{Evaluation of mobility}

- Timed Up and Go (TUG) test -This test is a clinical measure of dynamic balance and functional mobility for elderly individuals and consists of standing up from a chair without the aid of the arms, walking three meters with firm steps and at one's usual pace, turning around, walking back to the chair and sitting down again. The volunteer should have his/her back against the back of the chair at the beginning of the test and return to the same position at the end of the test. The test begins with a verbal cue ("go") and the time required to complete it is recorded from the verbal command to the moment the participant's back is once again in contact with the back of the chair. A total of 10 seconds or less indicates normal mobility; 11 to 20 seconds indicate frailty and more than 20 seconds indicates the need for assistance, further analysis and intervention. A score of 14 seconds or more suggests that the individual is prone to falls ${ }^{(18)}$. 
Besides the usual measure, data on acceleration and the time required to perform the simple phases of the TUG test will be collected using the G-WALK according Figure 2:

(1) TUG time: time (in seconds) required to perform the test;

(2) Phase 1: stand up

(a) Stand up phase: time (in seconds) required to stand up;

(b) Vertical acceleration of standing movement: maximum vertical acceleration (in $\mathrm{m} / \mathrm{seg}^{2}$ ) during rising phase;

(3) Phase 5: final $180^{\circ}$ rotation

(a) Final rotation phase: time (in seconds) required to execute final rotation;

(b) Mean velocity of final rotation: velocity (in degrees per second) required for final rotation;

(4) Phase 6: Sit down

(a) Sit down phase: time (in seconds) required to sit down;

(b) Vertical acceleration of sitting movement: vertical acceleration (in $\mathrm{m} / \mathrm{seg}^{2}$ ) during the sitting phase.

- Six-Minute Walk Test (6MWT) - The Six-Minute Walk Test (6MWT) will be performed on a flat track measuring 30 meters in length (with markings at each meter) and 1.5 meters in width without accompaniment following the guidelines of the American Thoracic Society(20). The participant will be instructed to walk as fast as possible for six minutes and will be encouraged with pre-established phrases at standardized one-minute intervals. The primary outcomes will be the distance (in meters) travelled in six minutes, the symmetry index, gait speed (m/s) and cadence (steps/minutes).
An Inertial Measurement Unit (IMU) will be used to measure spatiotemporal variables during the 6MWT. This device is composed of a 16-bit/axis triaxial accelerometer with multiple sensitivity ( $\pm 2, \pm 4, \pm 8$ and $\pm 16 \mathrm{~g}$ ), 13-bit triaxial magnetometer ( $\pm 1200 \mathrm{uT}$ ) and 16-bit/axis triaxial gyroscope with multiple sensitivity ( $\pm 250, \pm 500, \pm 1000$ and $\pm 2000 \%$ s). The inertial sensor has been validated by Bugané and colleagues ${ }^{(19)}$. The IMU (BTS G-WALK) has specific hardware (BTS G-SENSOR) and software (BTS G-STUDIO). The device will be attached to the participant's waist with a semi-elastic strap at the height of the L4-L5 inter-vertebral space. Acceleration will be collected on the three orthogonal anatomical axes (anterior-posterior, mid-lateral and vertical). Motion will be captured with a sampling frequency of $100 \mathrm{~Hz}$. The acceleration data will be transmitted via Bluetooth to a personal computer and processed using the specific software program (BTS G-STUDIO, version: 2.6.12.0). The following variables will be computed:

(1) Distance [m]traveled during the 6MWT;

(2) Symmetry index - between right and left sides of the gait cycle;

(3) Speed $[\mathrm{m} / \mathrm{s}]$ - mean instantaneous velocity within the gait cycle as the integration of acceleration;

(4) Cadence [steps/min].

Physiological variables will be collected before (baseline), during and after the 6MWT. Peripheral oxygen saturation $\left(\mathrm{SpO}_{2}\right)$ will be determined using a portable pulse oximeter (Nonin $^{\circledR}, \mathrm{GO}_{2}$ Achieve,USA). Heart rate (HR) will be determined using a pulse heart monitor (Polar ${ }^{\circledR}$, model 90440, Kempele, Finland). Blood pressure (BP) (Digital blood pressure monitor BP3AF1-3, China) will be determined before and after the test using a digital sphygmomanometer with the individual seated. Perceived exertion will be rated before and after

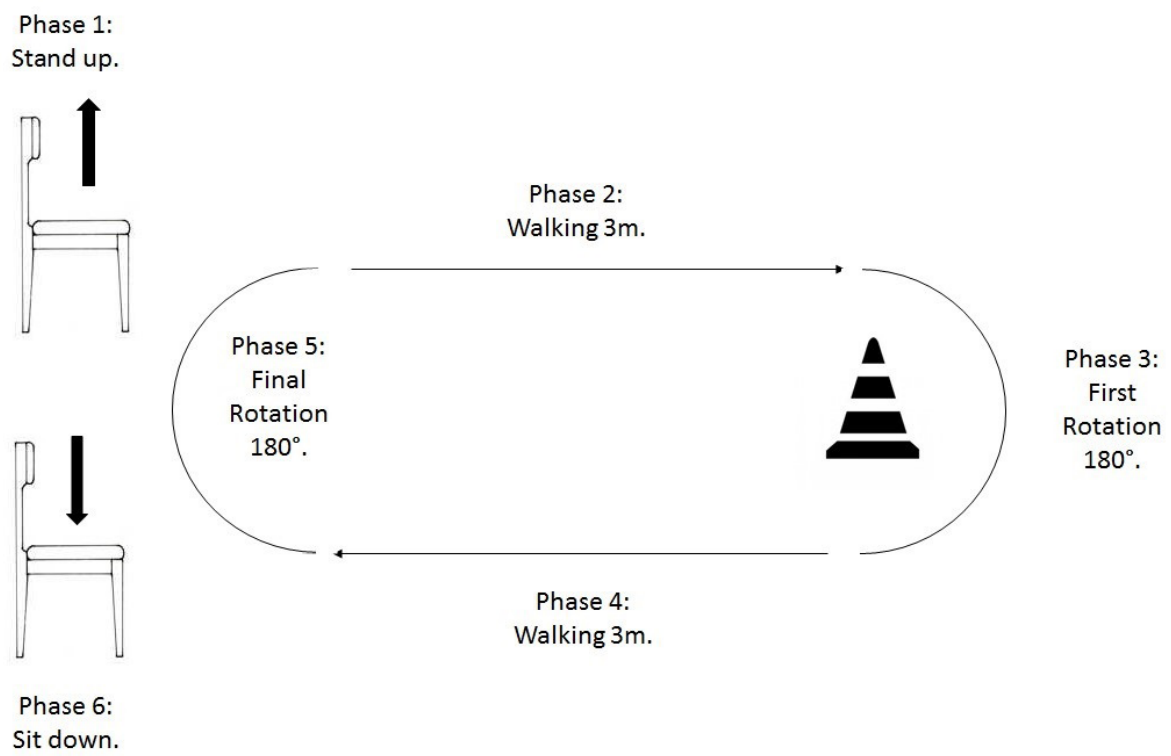

Figure 2. Timed Up and Go test (Adapted from Galli et al. ${ }^{(18)}$ ) 
the test using the modified Borg scale, which ranges from 0 (no effort) to 10 (extreme effort) ${ }^{(20,21)}$. During the 6MWT, the researcher will remain behind the participant and will accompany his/her pace.

\section{Intervention}

All procedures will be performed at the Parkinson Association Brazil in Sao Paulo. The individuals will participate in 20-minute training sessions three times a week for four weeks (total: 12 sessions) ${ }^{(22,23)}$. A specific aspect of the study will be the application of progressive, intensive gait training protocols that are similar to each other. Heart rate (HR), blood pressure (BP) and perceive exertion will be measure before, during (after 10 minutes of training) and after immediately after training. This procedure will be performed to ensure training intensity within the stipulated target range: $\mathrm{HR}$ between 60 and $70 \%$ of maximum HR ( 220 beats per minute - age $)^{(24,25)}$. BP will need to be below $200 / 100 \mathrm{~mm} \mathrm{Hg}$ and the modified Borg scale will be used for the determination of perceived exertion during the exercise session. Each group will receive the respective interventions, the main focus of which was physical fitness and motor coordination.

\section{Over ground gait training}

Training will be performed in a closed environment and will consist of routine exercises with controlled intensity. For such, hula hoops, cones, steps, balls, colored obstacles, horizontal ladders and stairs will be used. To execute the tasks, the participants walked past and/or around obstacles, went up and down a step, performed gait controlled by horizontal markings on the floor and pace controlled by the therapist's instructions.

\section{Treadmill training - TT}

Prior to the training sessions, the participants will be familiarized with the safe practice of the procedure. The treadmill is equipped with hand rails and a disconnection system to avoid falls. The training sessions will be adapted individually to each participant, who will be instructed to walk or run maintaining the HR within the target range. The participant will be instructed to maintain the correct body posture during the exercise and will always be under the strict supervision of the physiotherapist.

\section{Virtual reality gait training}

The participant will remain two to three meters in front of the motion sensor of the Kinect Xbox $360^{\mathrm{TM}}$ (Microsoft Corporation, Redmond, WA, USA). The multimedia projector projected the game Your Shape - Fitness Evolved 2012 - Run the World. This game was chosen based on its potential to stimulate motor coordination and improve physical fitness. The participant will simulate walking or running by lifting the knees in a stationary march, resulting in constant displacement of the center of gravity, involving symmetry, alternating actions and rhythm, which are essential to gait ${ }^{(26)}$.

\section{Statistical analysis}

Data distribution (normal or non-normal) will be tested using the Shapiro-Wilk test and equal variance will be tested using Levene's statistic. The data with normal distribution will be analyzed by using two-way ANOVA $(\alpha<0.05)$ and expressed as mean and standard deviation. For non-parametric data, Friedman's two-way ANOVA $(\alpha<0.05)$ will be used and the data will be expressed as median and interquartile range. All data will be organized and tabulated using the Statistical Package for Social Sciences - SPSS program, version 20.

\section{DISCUSSION}

This document offers a detailed description of a randomized, controlled, blind, clinical trial to determine the efficacy of virtual reality gait training in comparison to gait training performed on a treadmill and over ground with regard to balance and gait in individuals with PD. The results will be published and will contribute evidence regarding the use of different gait training methods for this population.

\section{TRIAL STATUS}

Patients are being recruited at the time of submission.

\section{Acknowledgments}

The authors are grateful to the Parkinson's Association Brazil.

\section{AUTHORS' CONTRIBUTIONS}

All authors made substantial contributions. Conceived and designed the experiments: GM RM PJ JL RL ND AD JJAK CO. Acquisition of data: GM RM JL RL ND ADEO CO. Interpretation of data: GMJJ AK MG LF EO CO. Contributed analysis tools: GMJJ PJ AK MG LF CO. Wrote the paper: GM RM JL PJ RL ND AD JJAK CO. Final approval of the version: GMJJ AK MG LF CO.

\section{CONFLICTS OF INTEREST}

The authors declare no conflict of interest.

\section{AUTHOR DETAILS}

${ }^{1}$ Doctoral and Master Program in Rehabilitation Sciences, Universidade Nove de Julho, São Paulo, SP, Brasil. Email: gilenouepa@yahoo.com.br, franco.renata@terra.com.br, jamilepalma@yahoo.com.br, robertalazzari@ hotmail.com, natycarvalho_fisio@hotmail.com, jrzaffalon@hotmail.com, arislanderlg@gmail.com. ${ }^{2}$ Departamento de Desporto, Universidade do Estado do Para, Altamira, PA, Brasil. ${ }^{3}$ Department of Electronic Information and Bioengineering, Politecnico di Milano and IRCCS San Raffaele Pisana, Roma, Italy. Email: anafrancisca.rozin@polimi.it, manuela.galli@polimi.it. ${ }^{4}$ Physiotherapy Department, Faculdade de Guairacá, Guarapuava, Paraná, Brasil. ${ }^{5}$ UNICENTRO, Guarapuava, Paraná, Brazil. Email: luiz_braun@hotmail.com. ${ }^{6}$ Physiotherapist, Associação Brasil Parkinson, São Paulo, SP. Email: erikaokamoto09@gmail.com

\section{REFERENCES}

1. Lord S, Godfrey A, Galna B, Mhiripiri D, Burn D, Rochester L. Ambulatory activity in incident Parkinson's: more than meets the eye? J Neurol. 2013; 260(12): 2964-2972.

2. Sanfelice, E. Parkinson's disease. Fisioter mov. 2004; 17(1): 11 - 24. 
3. Segev-Jacubovski O, Herman T, Yogev-Seligmann G, Mirelman A, Giladi N, Hausdorff J. The interplay between gait, falls and cognition: can cognitive therapy reduce fall risk? Expert Rev Neurother. 2011; 11(7): 1057-1075.

4. Lopes J, Lameira de Melo G, Lazzari R, Santos C, Franco de Moura R, Dumont $A$ et al. Measures used for the evaluation of balance in individuals with Parkinson's disease: a systematic review. Journal of Physical Therapy Science. 2016;28(6):1936-1942.

5. Mehrholz J, Friis R, Kugler J, Twork S, Storch A, Pohl M. Treadmill training for patients with Parkinson's disease. Cochrane Database of Systematic Reviews. 2009;(1): 1-33.

6. Petzinger G, Fisher B, Van Leeuwen J, Vukovic M, Akopian G, Meshul C et al. Enhancing neuroplasticity in the basal ganglia: The role of exercise in Parkinson's disease. Mov Disord. 2010; 25(S1): S141-S145.

7. Zettergren K, Franca J, Antunes M, Lavallee C. The effects of Nintendo Wii Fit training on gait speed, balance, functional mobility and depression in one person with Parkinson's disease. Med Health Sci J. 2011; 9(5): 18-24.

8. Buccello-Stout R, Bloomberg J, Cohen H, Whorton E, Weaver G, Cromwell R. Effects of Sensorimotor Adaptation Training on Functional Mobility in Older Adults. The Journals of Gerontology Series B: Psychological Sciences and Social Sciences. 2008; 63(5): P295-P300.

9. Mirelman A, Patritti B, Bonato P, Deutsch J. Effects of virtual reality training on gait biomechanics of individuals post-stroke. Gait \& Posture. 2010;31(4):433-437.

10. Melo G, Lopes J, Lopes Dumont A, de Moura R, Lazzari R, Junior J et al. P5: Analysis of the immediate effect of gait training with virtual reality on the parkinsonian walking. Gait \& posture 57(2017)203, 2017.

11. Galna B, Jackson D, Schofield G, McNaney R, Webster M, Barry G, et al. Retraining function in people with Parkinson's disease using the Microsoft kinect: game design and pilot testing. J Neuroeng Rehabil. 2014; 11(1): 60.

12. Mendes F, Pompeu J, Lobo A, da Silva K, Oliveira T, Zomignani A et al, et al. Motor learning, retention and transfer after virtualrealitybased training in Parkinson's disease - effect of motor and cognitive demands of games: a longitudinal, controlled clinical study. Physiotherapy. 2012; (98): 217223.

13. Ganesan M, Sathyaprabha T, Gupta A, Pal P. Effect of Partial WeightSupported Treadmill Gait Training on Balance in Patients With Parkinson Disease. PM\&R. 2014;6(1):22-33.

14. Miyamoto S, Lombardi Junior I, Berg K, Ramos L, Natour J. Brazilian version of the Berg balance scale. Brazilian Journal of Medical and Biological Research. 2004;37(9):1411-1421.
15. Martínez-Martín P, Gil-Nagel A, Gracia L, Gómez J, Martínez-Sarriés J, Bermejo F. Unified Parkinson's disease rating scale characteristics and structure. Movement Disorders. 1994;9(1):76-83

16. Hoehn MM, Yahr MD. Parkinsonism: onset, progression and mortality. Neurology. 1967; 17(5): 427-42.

17. Schenkman M, Clark K, Xie T, Kuchibhatla M, Shinberg M, Ray L. Spinal Movement and Performance of a Standing Reach Task in Participants With and Without Parkinson Disease. Physical Therapy. 2001;81(8):1400-1411.

18. Galli M, Kleiner A, Gaglione M, Sale P, Albertini G, Stocchi F, et al. Timed Up and Go test and wearable inertial sensor: a new combining tool to assess change in subject with Parkinson's disease after automated mechanical peripheral stimulation treatment. Int J Innov Technol. 2015; 4(11): 155-163.

19. Bugané F, Benedetti M, Casadio G, Attala S, Biagi F, Manca M, et al. Estimation of spatial-temporal gait parameters in level walking based on a single accelerometer: Validation on normal subjects by standard gait analysis. Computer Methods Programs in Biomedicine. 2012; 108(1): 129-137.

20. ATS - American Thoracic Society. ATS Statement: Guidelines For The SixMinute Walk Test. Amj Respir Care Med. 2005; 166: 111-117.

21. Enright P, McBurnie M, Bittner V, Tracy R, McNamara R, Arnold A, et al. The 6-min walk test: a quick measure of functional status in elderly adults. Chest. 2003; 123(2): 387-398.

22. Hirsch M, TooleT, Maitland C, Rider R. The balance training effects and high intensity resistance training in individuals with idiopathic Parkinson's disease. Arch Phys Med Rehabil. 2003; 84: 1109-1117.

23. Betker A, Szturm T, Moussavi Z, Nett C. Exercises based on the video game for balance rehabilitation: a pilot project. Arch Phys Med Rehabil. 2006; 87(8): 1141-1149.

24. Nadeau A, Pourcher E, Corbeil P. Effects of 24 wk of Treadmill Training on Gait Performance in Parkinson's Disease. Med Sci Sports Exerc. 2014; 46(4): 645-655.

25. Londeree $B$, Moeschberger $M$. Effect of age and other factors on maximal heart rate. Research quarterly for exercise and sport. 1982; 53(4): 297304.

26. Lazzari R, Politti F, Belina S, Collange Grecco L, Santos C, Dumont A, et al. Effect of Transcranial Direct Current Stimulation Combined With Virtual Reality Training on Balance in Children With Cerebral Palsy: A Randomized, Controlled, Double-Blind, Clinical Trial. J Mot Behav. 2016; 49(3): 329-336. 